\title{
Yeungnam University type drive-through (YU-Thru) coronavirus disease 2019 (COVID-19) screening system: a rapid and safe screening system
}

\author{
Wan Seok Seo ${ }^{1}$, Seong Ho Kim² ${ }^{2}$, Si Youn Song ${ }^{3}$, Jian Hur ${ }^{4}$, Jun Lee ${ }^{5}$, Sunho Choi ${ }^{6}$, Yoojung Lee , \\ Dai Seg Bai ${ }^{7}$ \\ ${ }^{1}$ Department of Psychiatry, Yeungnam University College of Medicine, Daegu, Korea \\ ${ }^{2}$ Department of Neurosurgery, Yeungnam University College of Medicine, Daegu, Korea \\ ${ }^{3}$ Department of Otorhinolaryngology-Head and Neck Surgery, Yeungnam University College of Medicine, Daegu, Korea \\ ${ }^{4}$ Department of Internal Medicine, Yeungnam University College of Medicine, Daegu, Korea \\ ${ }^{5}$ Department of Neurology, Yeungnam University College of Medicine, Daegu, Korea \\ ${ }^{6}$ Department of General Affairs, Yeungnam University Medical Center, Daegu, Korea \\ ${ }^{7}$ Department of Psychiatry, Yeungnam University Hospital, Daegu, Korea
}

Received: July 16, 2020

Revised: August 24, 2020

Accepted: August 31, 2020

Corresponding author:

Wan Seok Seo

Department of Psychiatry, Yeungnam University College of Medicine, 170 Hyeonchung-ro, Nam-gu, Daegu 42415, Korea

Tel: +82-53-620-3340

Fax: +82-53-629-0256

E-mail: sws3901@ynu.ac.kr
Active and prompt scale-up screening tests are essential to efficiently control the coronavirus disease 2019 (COVID-19) outbreak. The goal of this work was to identify shortcomings in the conventional screening system (CSS) implemented in the beginning of the outbreak. To overcome these shortcomings, we then introduced a novel, independently developed system called the Yeungnam University type drive-through (YU-Thru), and distributed it nationwide in Korea. This system is similar to the drive-throughs utilized by fast food restaurants. YU-Thru system has shortened the time taken to test a single person to 2-4 minutes, by completely eliminating the time required to clean and ventilate the specimen collection room. This time requirement was a major drawback of the CSS. YU-Thru system also reduced the risk of subjects and medical staff infecting one another by using a separate and closed examination system. On average, 50 to 60 tests were conducted per day when using the CSS, while now up to 350 tests per day are conducted with the YU-Thru system. We believe that the YU-Thru system has made an important contribution to the rapid detection of COVID-19 in Daegu, South Korea. Here, we will describe the YU-Thru system in detail so that other countries experiencing COVID-19 outbreaks can take advantage of this system.

Keywords: Conventional screening system; COVID-19; Rapid screening system; YU-Thru system

\section{Introduction}

Since the first cases of coronavirus disease 2019 (COVID-19) occurred in Wuhan, China in December 2019, the disease has rapidly spread worldwide [1]. A 35-year-old woman who had visited South Korea from Wuhan, China became the first case in South
Korea on January 20, 2020. As of July 31, 2020, a total of 14,305 COVID-19 cases have been reported in South Korea. The vast majority of new cases in this country have occurred in Daegu and the neighboring North Gyeongsang Province. In Daegu, the number of confirmed cases climbed rapidly after the first confirmed cases occurred on February 18, 2020. On March 30, Daegu reported

Copyright (C) 2020 Yeungnam University College of Medicine

This is an Open Access article distributed under the terms of the Creative Commons Attribution Non-Commercial License (http://creativecommons.org/licenses/by-nc/4.0/) which permits unrestricted non-commercial use, distribution, and reproduction in any medium, provided the original work is properly cited. 
6,624 cases of COVID-19, and North Gyeongsang Province reported 1,298 cases. Together, these accounted for $82.0 \%$ of all COVID-19 cases in South Korea at that time. In Daegu, the number of newly confirmed cases on February 19, 2020 was 15 . New cases exceeded 100 and 400 per day, by February 23rd and 27th, respectively, and the highest single-day spike to-date occurred on February 29, with 656 new cases. Since then, the number of new cases per day remained at approximately 400 to 600 and fell below 100 for the first time on March 10. On April 10, 2020, no newly confirmed cases of COVID-19 were reported in Daegu for the first time since the outbreak [2]. Meanwhile, coronavirus is spreading rapidly around the world, with 17,551,097 confirmed cases and 678,317 deaths as of July 31,2020 [3].

On March 12, 2020, the World Health Organization declared the COVID-19 outbreak a pandemic [4]. In order to contain the spread of infectious diseases, prompt detection of as many cases as possible through active laboratory testing, active quarantine of confirmed patients, systematic classification of patients according to the degree of symptoms, and initiation of step-by-step treatment strategies according to severity are crucial. Screening centers are isolated spaces that are designed to inspect and examine patients with COVID-19 symptoms such as fever and cough without contacting other patients or admitting patients into hospitals, thereby avoiding close contact with medical staff. Therefore, screening centers play a crucial role in preventing transmission by treating suspected COVID-19 patients separately from other patients.

In South Korea, screening centers have been operating throughout the nation since January 24, 2020 [5]. The Yeungnam University Hospital (YU) set up a conventional screening system (CSS) on February 17, 2020, under the guidance of the Korea Centers for Disease Control and Prevention (KCDC).

\section{Brief overview of the conventional screening system and its' limitations}

According to the KCDCs' guidelines for screening centers, a screening center should be located outside the hospital. Procedures should be implemented inside negative-pressure rooms/ tents that can be separated from other areas [6]. In particular, sample collection should be performed solely in negative-pressure tents. However, the CSS had several limitations. First, a number of patients were exposed to cold weather while they were waiting for their tests. COVID-19 cases were rapidly rising in Daegu in mid-February, and temperatures had dropped to $-5^{\circ} \mathrm{C}[7]$. Consequently, many people were more likely to have respiratory diseases such as common colds, as well as experience the inconvenience of waiting in long lines. In the CSS, a long line often had people wait- ing to be tested for up to 5 hours. Second, infectious diseases were communicable to the crowd of individuals waiting in line to be tested. Under the CSS systems' initial testing conditions, the positive case rate in screening centers was approximately $5 \%$, which was quite high [8]. Therefore, it was likely that COVID-19 was transmitted from confirmed patients to other patients who were in close contact. In addition, under the CSS procedures, there was a possibility of medical staff being infected by confirmed COVID-19 patients. Although it was recommended that medical staff wear goggles and maintain a distance of more than $2 \mathrm{~m}$ from subjects, as well as use level $D$ clothing and a face shield if they were within $2 \mathrm{~m}$ of subjects, the risk of infection could not be ruled out [6]. Third, the amount of time required to clean and ventilate test rooms after sampling was considerable: with the CSS procedure, at least 30 minutes was required to achieve $>99 \%$ airborne-contaminant removal efficacy, which is incompatible with the large-scale screening required by COVID-19 [9].

\section{Design and launch of the Yeungnam University type drive-through screening system}

Yeungnam University Hospital (YU), one of the major university hospitals in Daegu, operated a CSS beginning on February 17, 2020. However, as the number of newly confirmed patients in Daegu rose, more people visited the screening centers to undergo testing. Once the number of tested subjects exceeded 20 per day, the CSS was unable to meet the testing requirements, and individuals were irritated by the failure of appropriate screening tests. Additionally, early in the COVID-19 outbreak, Daegu underwent a critical medical problem, as well as the inefficient CSS function. At one point, four of five emergency centers of university hospitals in Daegu were closed at the same time due to exposure to COVID-19. From February 19 to 22, YU was also closed three times, for a total of 100 hours of shutdown. We then realized the need for a more efficient screening system.

YU initially solved the CSS problem of subjects being exposed to cold weather. To prevent subjects from contracting infectious respiratory diseases including common colds, and from transmitting diseases to each other, we requested that patients remain in their cars while awaiting testing. Individuals were then notified of their turn through a phone call. Next, we considered the issue of how to decrease the time required to clean and ventilate the negative-pressure tents. The solution we developed was to ensure that individuals did not leave their cars throughout the testing procedure, from the stand-by period to exiting the testing center. We considered a patient's car to be a closed space in which there was no contact 
with other patients, and therefore no risk of infecting other individuals. In addition, there was no need to ventilate and clean the car as tested individuals did not share cars with each other. In addition to these improvements, we also minimized contact between the medical staff and subjects by using shipping containers as medical staff examination rooms. Medical staff and subjects communicated through a two-way microphone and speaker. Upon receiving news from the government concerning system insurance coverage, we launched the Yeungnam University type drive-through (YU-Thru) system. Overall, the YU-Thru system consists of four steps, with details provided below.

\section{The four steps of Yeungnam University type drive-through system with a detailed description of each step}

The goal of YU-Thru system was to simplify the testing procedure as much as possible to maximize the number of individuals that could be tested per day. The YU-Thru system process consists of four steps; registration, medical examination, payment, and sample collection. The YU-Thru system occupied approximately $1,527 \mathrm{~m}^{2}$ of parking lot space (Fig. 1). Individuals were requested to drive by themselves and to have one-person-per-car if possible, as having two or more people in one car could encourage transmission between the cars' occupants. However, if the individuals were elderly or could not drive by themselves (e.g., infants, children, or patients with psychiatric disorders such as schizophrenia, severe mania, or depression), caregivers were allowed to drive with them.
Of the four steps, three were performed almost entirely inside the shipping containers. In step 4, sample collection was performed outside the container. The staff and medical personnel involved in steps 1,3 , and 4 were equipped with level D protection, including KF94 or N95 grade face masks and face shields. The doctors in step 2 wore only KF94 or N95 grade face masks.

\section{Step 1: registration and creating a hospital number}

First, each person to be tested placed their identification (ID) card into the scanner. Then the ID card was scanned into the computer, as shown in Fig. 2. The YU registration number was automatically created when the staff member entered the patient's date of birth and social security number, as displayed on the computer monitor. For non-nationals, the YU registration number was created using the patient's alien registration card; however, the remainder of the process remained the same. For non-nationals who were not registered in South Korea (e.g., tourists or illegal aliens), the YU registration number was created from passport numbers. The staff then recorded the home address and cell phone number of subjects using the two-way microphone and speaker system. It was important to enter the correct cell phone number into the computer as test results were texted. At the same time, a separate staff member used a noncontact forehead thermometer to measure the subject's temperature. Minor exposure to infected patients may have occurred during this procedure. Although two staff members were recommended overall for step 1 , it was possible to complete the registration and temperature testing with one staff member performing

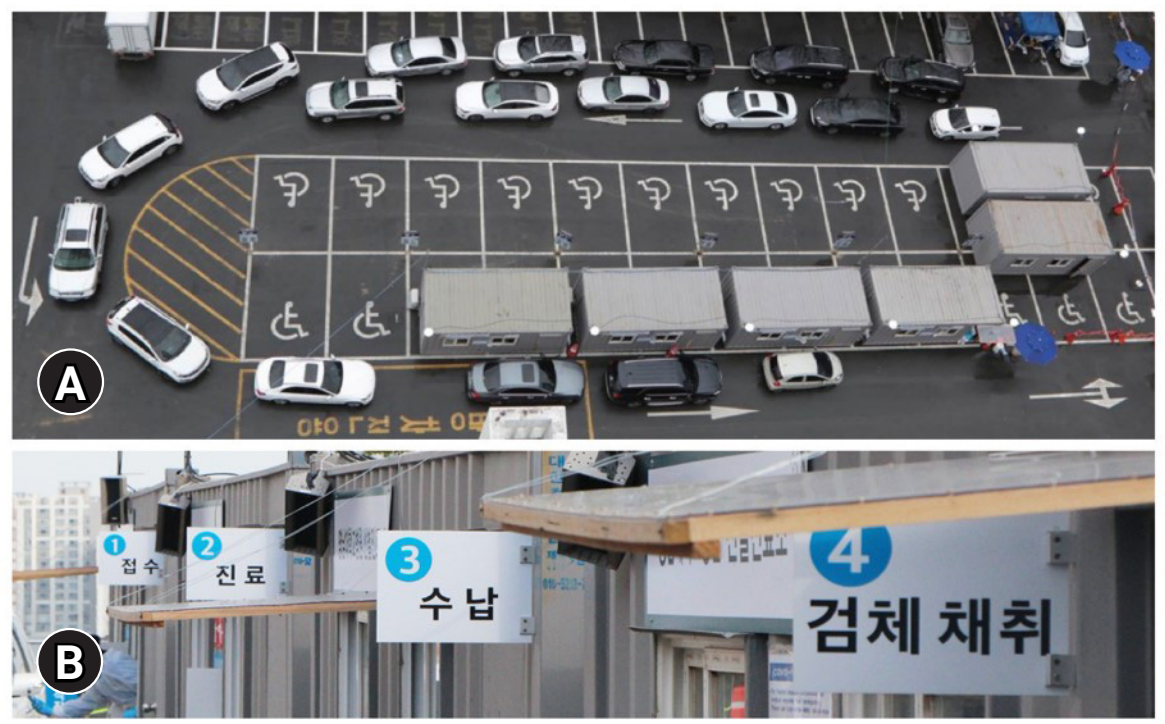

Fig. 1. (A) Aerial photography of the Yeungnam University type drive-through (YU-Thru) system. (B) Four steps of the YU-Thru system; (1) registration, (2) medical examination, (3) payment, and (4) specimen collection. 

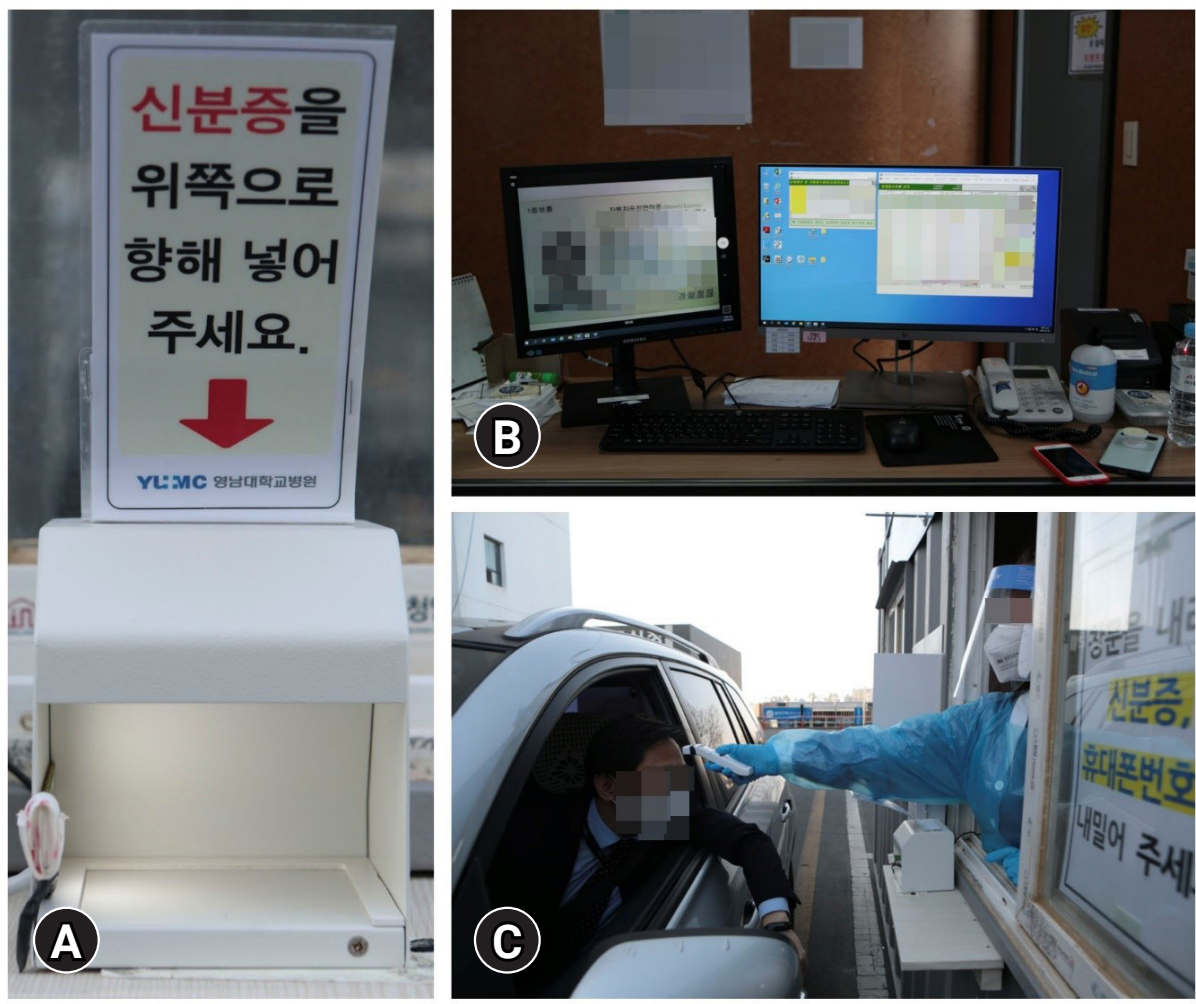

Fig. 2. Step 1. (A) Identification (ID) card scanner (description in Korean: "Please insert the ID card with photo-side up."). (B) A scanned image of the ID card on a computer monitor. (C) A noncontact forehead thermometer was used to measure an individuals' body temperature.

both procedures (Fig. 2).

\section{Step 2: medical examination by a physician}

For this step in our study, physicians from YU, volunteer doctors in Daegu, and public health doctors in South Korea participated. Physicians thoroughly checked the individuals' symptoms including fever, sore throat, rhinorrhea, nasal congestion, cough, phlegm, and disturbances of smell and taste. They also identified whether the individuals had underlying diseases including lung disease, heart disease, diabetes, hypertension, kidney disease, and cancer. In addition, doctors checked whether there was contact with previously COVID-19 confirmed patients, whether subjects had attended a particular religious meeting, or whether they had visited the hospital located in an infection cluster.

After this screening, the doctors decided whether the individual needed to be tested, whether a nasal and throat or sputum test should be performed, and whether insurance would cover the testing. The test was then prescribed, and if covered by insurance, the physician completed a document called an "infectious patient report" online and sent it to the KCDC. The doctors asked the individuals questions through two-way microphones, and there was no contact between them (Fig. 3). Therefore, the risk of coronavi-

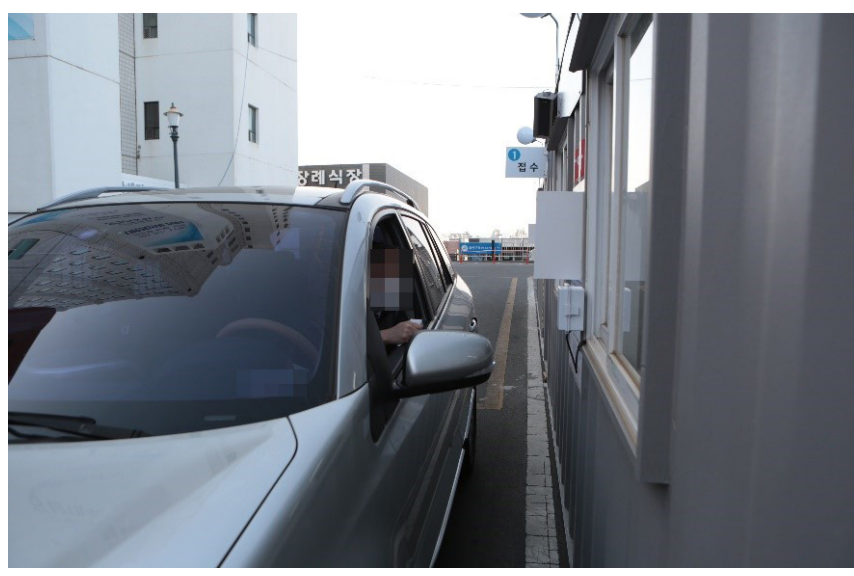

Fig. 3. A two-way microphone is used during communication between an individual and a doctor. The doctor and individual view one another through the window and talk via the microphone.

rus contamination was considered to be nonexistent.

\section{Step 3: payment}

In this step, the subject paid the registration, examination, and inspection fees. Computers installed with payment systems and card readers were set inside a shipping container. Although paying by 
credit or debit cards was preferred, cash payments were also permitted. Although paying by card is superior in preventing infection, some individuals requested to pay with cash. As some contact was inevitable during this process, there was a risk of contamination by exchanging cards, cash, and/or receipts (Fig. 4).

\section{Step 4: sample collection}

After the individual paid the required fees sample labels were printed from the computer. Medical staff then brought labeled sample tubes to the individual's car and cross-checked the information. The individual was instructed to stay in the car throughout the process. To obtain a throat swab, the individual lowered their face mask and opened their mouth. To obtain a nasal swab, the individual wore a face mask and kept their mouth closed. To collect sputum samples, the individual produced phlegm by coughing while wearing a face mask, and then spit the phlegm into a tube while the car's windows were closed. The individual then opened the cars' window and gave the tube to the medical staff. This procedure was the greatest source of contamination throughout the process. Medical staff were equipped with level D protection clothing, an N95 grade mask, and face shields, as well as apron gowns (Fig. 5). Apron gowns were replaced between each test.

\section{Results and advantages of the Yeungnam University type drive-through screening system}

The YU-Thru system had several advantages over CSS. First, the YU-Thru system allowed us to dramatically reduce the time required to clean and ventilate testing areas. As a result, we were able to perform more tests with the YU-Thru system than with CSS. When operating a CSS, the number of tests performed in a day was 50, which

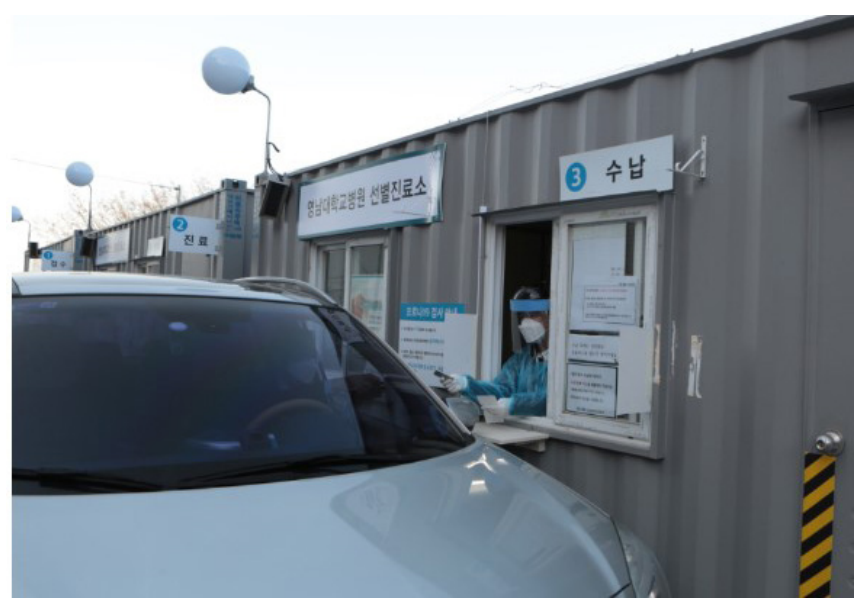

Fig. 4. Payment using a credit card. increased to an average of 149 cases per day until March 30. Meanwhile, 339 tests were conducted on March 1, 2020 (Fig. 6). The time required to test a single individual was approximately $30 \mathrm{~min}$ utes under the CSS; however, this was reduced to approximately 2 to 4 minutes under the YU-Thru system. The main reason for this improvement was that there was no need to clean and ventilate the examination rooms. As shown in Fig. 6, the number of tests met the demand as the number of confirmed cases in Daegu increased. Since March 14, the number of tests has gradually declined. This likely reflects a decrease in cases in Daegu from 100 per day on March 12, after which a decrease in required testing was observed. Second, the YU-Thru system improved the safety of both the individuals to be tested and the medical staff. A total of 61 doctors and 89 medical and nonmedical hospital staff participated in the system from February 26 to March 30; however, none were confirmed as positive for COVID-19. Although we could not entirely rule out infections using this system, the YU-Thru system utilized segregated spaces including specimen collection rooms, unlike the shared specimen collection rooms in CSS, which posed a risk of infection. As a result, the possibility of transmission from one subject to another was completely eliminated, and we prevented or dramatically reduced the infection of medical staff by tested individuals. Third, YU-Thru system can be readily constructed. Cities without parking lots adjacent to hospitals can be easily modified to become testing sites. If the testing process of YU-Thru system is installed in an underground parking lot or closed space, the lack of ventilation may increase vulnerability to infection, and therefore caution is necessary. Consequently, we recommend installing the YU-Thru process outdoors where natural ventilation is possible. For construction, our test sites required approximately $1,527 \mathrm{~m}^{2}$ of space, four shipping containers, computers, computer network systems, and several personnel. In addition, flexible expansion was

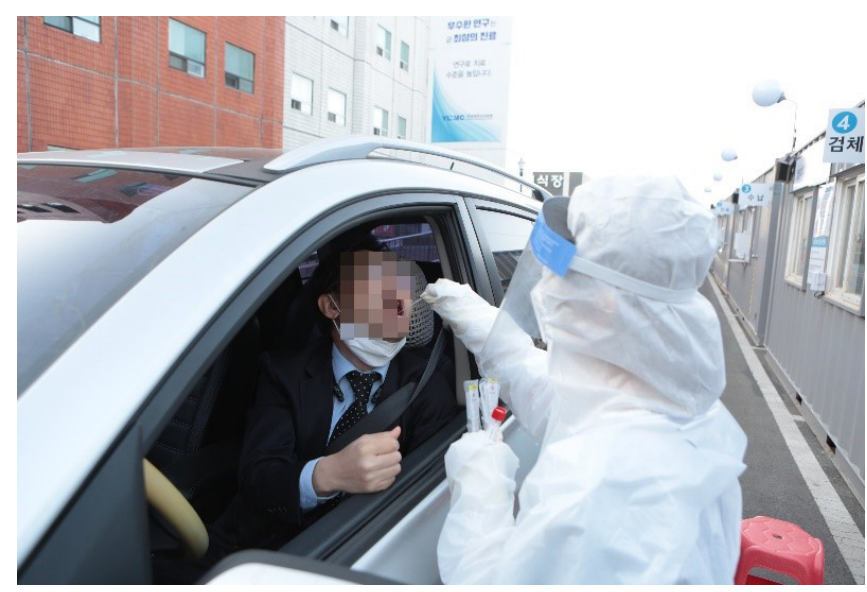

Fig. 5. Throat swab procedure. 


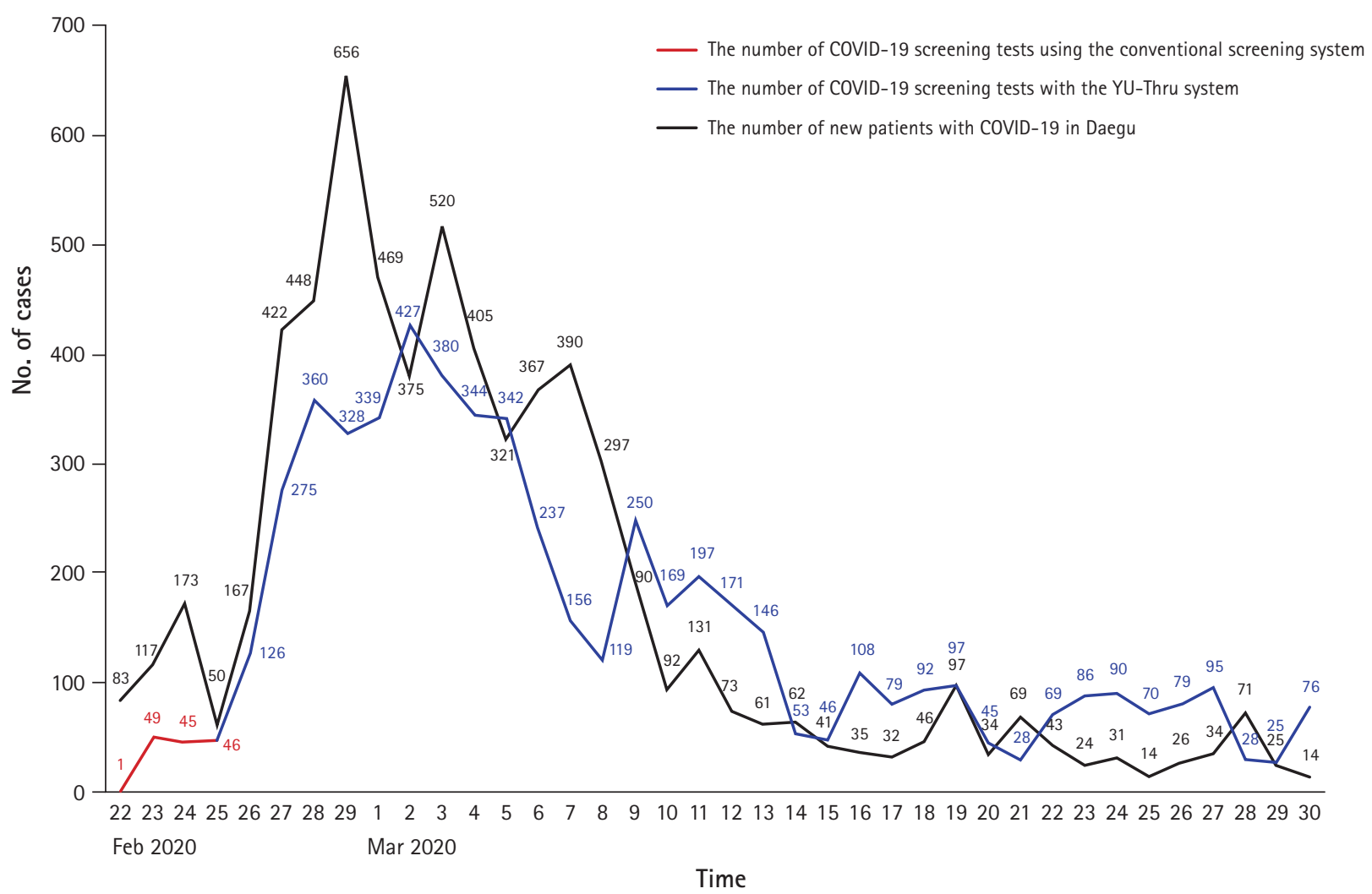

Fig. 6. A comparison of the number of tests achieved using the conventional screening system and the Yeungnam University type drivethrough (YU-Thru) system. Red, the number of tests using the conventional screening system; blue, the number of tests using the YUThru system; black, the number of newly confirmed cases in Daegu, South Korea. COVID-19, coronavirus disease 2019.

possible depending on the demand. If there was a growing number of newly confirmed cases and the need for screening tests increased, additional YU-Thru system sites were constructed in nearby parking spaces. Moreover, only 1 or 2 days days were required to install the YU-Thru system.

\section{Limitations of the Yeungnam University type drive-through screening system and its'specific concerns}

Although the YU-Thru system has enabled faster and safer screening tests compared with the CSS, and none of the staff were confirmed to be COVID-19 positive, additional complementation is necessary to further reduce the chances of infection. First, during the payment step (step 3), if the subject swipes or inserts their own credit card and takes their receipt, the process is completely contactless and the possibility of contamination is zero. Similarly, using noncontact forehead thermometers in step 1 carries a nonexistent risk for infection. Second, it is necessary to find ways to reduce the possibility of infection in passengers sharing the same car with individuals who may be infectious. In this study, most individuals drove by themselves; however, elderly, disabled (e.g., dementia or autism spectrum disorder), or children were often accompanied by caregivers and the possibility of infection transmission could not be ruled out. A system to minimize the risk of infection in these people is necessary.

\section{Conclusion}

In order to flatten the curve of the worldwide spread of COVID-19, it is crucial to identify confirmed patients through massive screening, and to initiate preemptive isolation and active treatment measures against positively confirmed patients. To achieve this goal, a safe and efficient screening system such as the YU-Thru system is necessary. We have introduced the YU-Thru system not only to a number of health care facilities and hospitals in South Korea but also to numerous countries around the world [10-13]. We hope that many additional countries will implement and properly utilize the YU-Thru system in the fight against COVID-19. 


\section{Acknowledgments}

\section{Conflicts of interest}

No potential conflict of interest relevant to this article was reported.

\section{Author contributions}

Conceptualization: WSS, SHK, SYS, JH, JL, SC, DSB; Data curation: SHK, SC, DSB; Formal analysis, Methodology, Investigation, and Software: DSB; Resources: SC; Writing-original draft: WSS, JH, YL; Writing-review \& editing: WSS, SYS, JH, JL, YL.

\section{ORCID}

Wan Seok Seo, https://orcid.org/0000-0002-5122-5360

Si Youn Song, https://orcid.org/0000-0001-6642-8841

Jian Hur, https://orcid.org/0000-0003-3219-8368

Jun Lee, https://orcid.org/0000-0001-8643-0797

Sunho Choi, https://orcid.org/0000-0001-5270-2467

Yoojung Lee, https://orcid.org/0000-0002-7924-0247

Dai Seg Bai, https://orcid.org/0000-0002-6907-6201

\section{References}

1. Wang C, Horby PW, Hayden FG, Gao GF. A novel coronavirus outbreak of global health concern. Lancet 2020;395:470-3.

2. CoronaBoard. COVID-19 dashboard [Internet]. CoronaBoard; 2020 [cited 2020 Jul 7]. https://coronaboard.kr/.

3. Corona Virus Resource Center, Johns Hopkins University \& Medicine. COVID-19 dashboard by the Center for Systems Science and Engineering (CSSE) at Johns Hopkins University (JHU) [Interent]. Baltimore (MD): Johns Hopkins University \& Medicine; 2020 [cited 2020 Jul 7]. https://coronavirus.jhu. edu/map.html.

4. World Health Organization Regional Office For Europe. WHO announces COVID-19 outbreak a pandemic [Internet]. Copenhagen: WHO Regional Office for Europe; 2020 [cited 2020 Jul 7]. http://www.euro.who.int/en/health-topics/health-emergencies/coronavirus-covid-19/news/news/2020/3/who-announces-covid-19-outbreak-a-pandemic.

5. Korean Society of Infectious Diseases; Korean Society of Pediatric Infectious Diseases; Korean Society of Epidemiology; Korean Society for Antimicrobial Therapy; Korean Society for
Healthcare-associated Infection Control and Prevention; Korea Centers for Disease Control and Prevention. Report on the epidemiological features of coronavirus disease 2019 (COVID-19) outbreak in the Republic of Korea from January 19 to March 2, 2020.J Korean Med Sci 2020;35:e112.

6. Central Disaster Management Headquarters, Central Diease Control Headquarters. Guidance for operation of COVID-19 screening centers. Released on February 21, 2020. Sejong (KR): Ministry of Health and Welfare; 2020.

7. AccuWeather. Monthly weather forecast. February 2020 in Daegu, South Korea [Internet]. State College (PA): AccuWeather; 2020 [cited 2020 Jul 7]. https://www.accuweather. com/en/kr/daegu/223347/february-weather/223347.

8. Jung SM, Akhmetzhanov AR, Hayashi K, Linton NM, Yang Y, Yuan B, et al. Real-time estimation of the risk of death from novel coronavirus (COVID-19) infection: inference using exported cases. J Clin Med 2020;9:523.

9. Kohn WG, Collins AS, Cleveland JL, Harte JA, Eklund KJ, Malvitz DM, et al. Guidelines for infection control in dental healthcare settings: 2003. MMWR Recomm Rep 2003;52(RP-17): $1-61$.

10. Sophie Lewis. CBS News: South Korea is using fast food-style drive-thrus to test for coronavirus [Internet]. New York: CBS Interactive Inc.; March 6, 2020 [cited 2020 Jul 7]. https://www. cbsnews.com/news/coronavirus-south-korea-drive-thru-testcovid-19/.

11. Reuters. South Korea runs 'drive-thru' virus testing in Daegu [Internet]. London: Reuters; March 4, 2020 [cited 2020 Jul 7]. https://uk.reuters.com/video/watch/south-korea-runs-drivethru-virus-testin-id689445178.

12. Nikkei staff writers. Coronavirus: Week of Mar. 1 to Mar. 7, confirmed cases top 100,000 worldwide [Internet]. Tokyo: Nikkei Asian Review; March 2, 2020 [cited 2020 Jul 7]. https://asia. nikkei.com/Spotlight/Coronavirus/Coronavirus-Free-to-read/ Coronavirus-Week-of-Mar.-1-to-Mar.-7-confirmed-cases-top100-000-worldwide.

13. AFP. COVID-19: China reports fall in cases, South Korea death toll hits 32 [Internet]. Beijing: The Hindu; March 4, 2020 [cited $2020 \mathrm{Jul}$ 7]. https://www.thehindu.com/news/international/covid-19-china-reports-fall-in-cases-south-korea-death-tollhits-32/article30978782.ece\#. 
